CVL IMPAIRS IMPLICIT LEARNING

In press, Cortex (Feb. 2021)

\title{
Simulated central vision loss impairs implicit location probability learning
}

\author{
Douglas A. Addleman ${ }^{1,2}$ \\ Gordon E. Legge ${ }^{2}$ \\ Yuhong V. Jiang ${ }^{2}$ \\ 1. Dartmouth College \\ 2. University of Minnesota
}

\begin{abstract}
Some eye diseases, especially macular degeneration, can cause central vision loss (CVL), impairing goal-driven guidance of attention. Does CVL also affect implicit, experience-driven attention? We investigated how simulated central scotomas affected young adults' ability to prioritize locations frequently containing visual search targets (location probability learning). Participants searched among distractor letter ' $L$ 's for a target ' $T$ ' that appeared more often in one screen quadrant than others. To dissociate potential impairments to statistical learning of target locations and attentional guidance, two experiments each included search with and without simulated scotomas. Experiment 1 successfully induced probability learning in a no scotoma phase. When participants later searched both with and without simulated scotomas, they showed persistent, statistically equivalent spatial biases in both no-scotoma and scotoma search. Experiment 2 trained participants with a central scotoma. While Experiment 1's participants acquired probability learning regardless of their self-reported awareness of the target's location probability, in Experiment 2 only aware participants learned to bias attention to the high probability region. Similarly, learning with a scotoma affected search with no scotoma in aware but not unaware participants. Together, these results show that simulated central vision loss interferes with the acquisition of implicitly learned location probability learning, supporting a role of central vision in implicit spatial attentional biases.
\end{abstract}

Keywords: visual attention; selection history; central vision loss; visual search; location probability learning

\section{Introduction}

Central vision loss (CVL) affects over 170 million people worldwide, most commonly resulting from age-related macular degeneration (Pennington \& Deangelis, 2016). Loss of central vision impairs critical real-world behaviors that benefit from high-acuity vision, such as reading and driving (Cheung \& Legge, 2005). Such impairments result not only from the loss of highacuity central vision, but also a disruption in eye movements (Caldani, Chatard, Wiener-Vacher, \& Bucci, 2019; Thibaut, Tran, Szaffarczyk, \& Boucart, 2017). These impairments are thought to underlie deficits in goal-driven attention in patients with CVL, manifested as slower visual search especially in more naturalistic search tasks (for a review, see Crabb \& Taylor, 2017). But does CVL also affect implicit, experience-driven attention?

Recent research has shown that eye movements and visuospatial attention are affected not only by top-down goals, but also by previous experience, for example via "selection history" effects (Awh, Belopolsky, \& Theeuwes, 2012). Repeating the visuospatial context within which 
a target appears facilitates search, leading to contextual cueing (Chun \& Jiang, 1998). Likewise, search is faster if targets frequently appear in one region of the visual field, leading to location probability learning (Druker \& Anderson, 2010; Geng \& Behrmann, 2002; Miller, 1988). Contextual cueing and location probability learning both induce strong attentional biases even when participants lack awareness of cueing (Colagiuri \& Livesey, 2016; Jiang, Sha, \& Sisk, 2018). The implicit nature of these effects allow them to facilitate search in many conditions that impair goal-driven attention, including during dual-task processing and healthy aging (Jiang, Sisk, \& Toh, 2019; Twedell, Koutstaal, \& Jiang, 2017; Won \& Jiang, 2015). These findings raise the possibility that unlike goal-driven attention, implicitly guided attention may be preserved under conditions of central vision loss.

Studies on contextual cueing, however, have found that CVL can interfere with implicit guidance of attention. Both in patients with age-related macular degeneration (Geringswald, Herbik, Hoffmann, \& Pollmann, 2013) and in healthy adults searching with a simulated central scotoma (Geringswald, Baumgartner, \& Pollmann, 2012; Geringswald \& Pollmann, 2015), impaired central vision prevented the expression of contextual cueing. However, these results may not generalize to other forms of experience-driven attention (e.g., location probability learning). Contextual cueing requires not just implicit guidance to a target's location, but also associative learning connecting repeated contexts to a target location. Probability learning, on the other hand, depends on learning probabilities of targets appearing in different regions regardless of context as well as attentional guidance to locations of high target probability. Central vision loss may impair only certain kinds of implicitly guided attention that benefit from a wholly intact visual field or other features impaired by CVL. Despite location probability learning's prevalence in everyday situations (e.g., finding traffic signals in consistent locations in one's visual field) and its importance in recent theories of spatial attention (Jiang, 2018), to date there have been no prior studies of the effects of CVL on location probability learning.

Previous findings could predict either intact or impaired location probability learning following central vision loss. On the one hand, probability learning is intact in other populations with impairments to goal-driven attention, including Parkinson's patients (Sisk, Twedell, Koutstaal, Cooper, \& Jiang, 2018), children with Autism Spectrum Disorders (Jiang, Capistrano, Esler, \& Swallow, 2013), and healthy older adults (Twedell et al., 2017). On the other hand, certain theories suggest that probability learning involves mechanisms of attentional guidance that may be disrupted by CVL. Specifically, location probability learning may reflect an attentional habit to a learned region of space, such that participants learn the appropriate direction to shift attention - often modeled as a vector directing spatial attention from fixation rather than an abstract representation of target locations in a Cartesian map (Jiang, 2018). If probability learning involves oculomotor habits, introduction of simulated CVL is likely to impair learning. Due to differences inherent to searching with foveal as opposed to peripheral vision, the introduction of CVL may interfere with the development of an appropriate search habit, impairing the acquisition of location probability learning. Even after probability learning has been acquired without CVL, introducing CVL may alter oculomotor behavior, interfering with the expression of location probability learning. This is especially plausible because participants with healthy vision will continue to habitually fixate using the fovea while learning to search with a central scotoma (Geringswald et al., 2012), a habit that takes many hours of training to overcome (Kwon, Nandy, \& Tjan, 2013). Investigating the effects of simulated CVL on location probability learning may clarify its cognitive and neural mechanisms. 
Here, we simulated central vision loss in healthy young adults and used behavioral and eye tracking measures to investigate location probability learning. Participants completed a Tamong-L search task that induced location probability learning by making targets more likely to occur in one visual quadrant during a training phase. We then tested the preservation of probability learning after the target's location probability became equiprobable. Experiment 1 trained participants without CVL to induce location probability learning. We then introduced CVL in the testing phase to assess whether expression of learning to attend to the highprobability locations is disrupted by CVL (does CVL impair attentional guidance?). Experiment 2 tested how CVL affected the acquisition of location probability learning (does CVL impair statistical learning?). We trained participants with CVL and then removed the CVL in the testing phase. Together, these experiments inform a theoretical understanding of oculomotor and attentional impairments due to CVL as well as addressing outstanding questions about the level of guidance involved in location probability learning. They also aim to inform future applied research on location probability learning in patients with age-related macular degeneration and its potential role in overcoming known deficits in goal-driven and other forms of attention (Crabb \& Taylor, 2017).

\section{Method}

Participants. College students from the University of Minnesota community completed this study after providing informed consent and were compensated for their time with either extra course credit or $\$ 10 /$ hour. All participants had normal or corrected-to-normal visual acuity and signed an informed consent form prior to participating. Each participant took part in one experiment. The University of Minnesota Institutional Review Board provided ethics approval for these experiments. We report how we determined our sample size, all data exclusions, all inclusion/exclusion criteria, whether inclusion/exclusion criteria were established prior to data analysis, all manipulations, and all measures in the study. Study design and analyses were not pre-registered.

Data were collected until we obtained usable data from 24 participants in each of Experiments 1 and 2. The sample size - 24 - was pre-determined to be comparable to or larger than previous studies on location probability learning (Jiang, Swallow, Rosenbaum, \& Herzig, 2013). Data from 7 additional participants were excluded based on criteria determined prior to data analysis, 6 in Experiment 1 and 1 in Experiment 2, due to poor eye tracking quality ( $\mathrm{N}=3$ ), computer problems $(\mathrm{N}=2)$, and accuracy during scotoma search below 2 standard deviations from the mean scotoma search of all participants in that experiment $(\mathrm{N}=2)$.

The 24 participants in Experiment 1 included 17 women and 7 men, with a mean age of 20.4 years (range: 18-21). The 24 participants in Experiment 2 included 16 women and 8 men, with a mean age of 18.9 years (range: 18-21).

Equipment. Participants completed the study with their head in a chinrest. Viewing distance was $90 \mathrm{~cm}$. The experiment was implemented using MATLAB and Psychtoolbox (Kleiner, Brainard, \& Pelli, 2007), as well as EyeLink software (SR Research). Stimuli were presented on a 19-inch CRT monitor with a vertical refresh rate of $100 \mathrm{~Hz}$ and resolution of $1024 \times 768$ pixels.

Eye tracking was conducted using EyeLink 1000 Plus eye tracker (SR Research, Mississauga, Canada) with a sampling rate of 1,000 Hz. Eye position was calibrated using a 9point calibration process prior to the experiment, with drift verification prior to each trial. A researcher remained in the room to monitor eye tracking quality and re-calibrated the eye tracker 
as needed throughout the experiment. The eye tracker was used to present a gaze-contingent scotoma in some trials. This was done using the identified fixation location from the eye tracker and was updated at the refresh rate of the monitor $(100 \mathrm{~Hz})$.

To ensure that any lag between actual eye movements and updating of the scotoma location did not provide useful central vision, we tested 9 other participants in an experiment using a procedure described by Geringswald et al. (2013). Participants reported the gap direction of a small C stimulus (up, down, left, or right) while searching either with or without a simulated central scotoma (which was $6.7^{\circ}$ in diameter as in the main experiments). At 2.75 minutes of arc, the target's gap was large enough to be detected foveally but too small to be detected with peripheral vision, so participants should perform well in no-scotoma search but at chance (i.e., $25 \%$ ) in scotoma search. In contrast, if our simulation let useful information leak into central vision due to the temporal lag in presentation of the scotoma, performance should have been above chance in scotoma search. A bootstrapped standard error analysis showed that all 9 participants were at chance during scotoma search (mean accuracy was $26 \%, \mathrm{SE}=1 \%$ ) and were significantly more accurate during no-scotoma search (mean accuracy was $91 \%, \mathrm{SE}=3 \%$ ). Thus, the eye tracker effectively prevented useful visual processing in the region of the scotoma.

Stimuli. Figure 1 shows a sample search trial during scotoma search; a video depicting several trials is available at https://osf.io/4g7vc/. During search both with and without the central scotoma, search items were white letters $\left(1^{\circ} \times 1^{\circ}\right)$ presented within black circles $\left(1.4^{\circ}\right.$ diameter $)$. To encourage the use of overt eye movements to complete the task, stimuli were presented at the same size regardless of eccentricity. Each trial contained 12 items presented at locations on an invisible $10 \times 10$ grid $\left(12.5^{\circ} \times 12.5^{\circ}\right)$, with the constraint that 3 items appeared in each screen quadrant. To make the search task more realistic, the array of letters was presented on top of a natural scene, randomly drawn from 144 possible scenes from the SUN397 database (Xiao, Hays, Ehinger, Oliva, \& Torralba, 2010).

The scotoma was a black circle displayed on top of all other stimuli at the location of fixation approximately $6.7^{\circ}$ in diameter (analogous to those used in past research, e.g., Kwon et al., 2013). Its location was updated at $100 \mathrm{~Hz}$. 


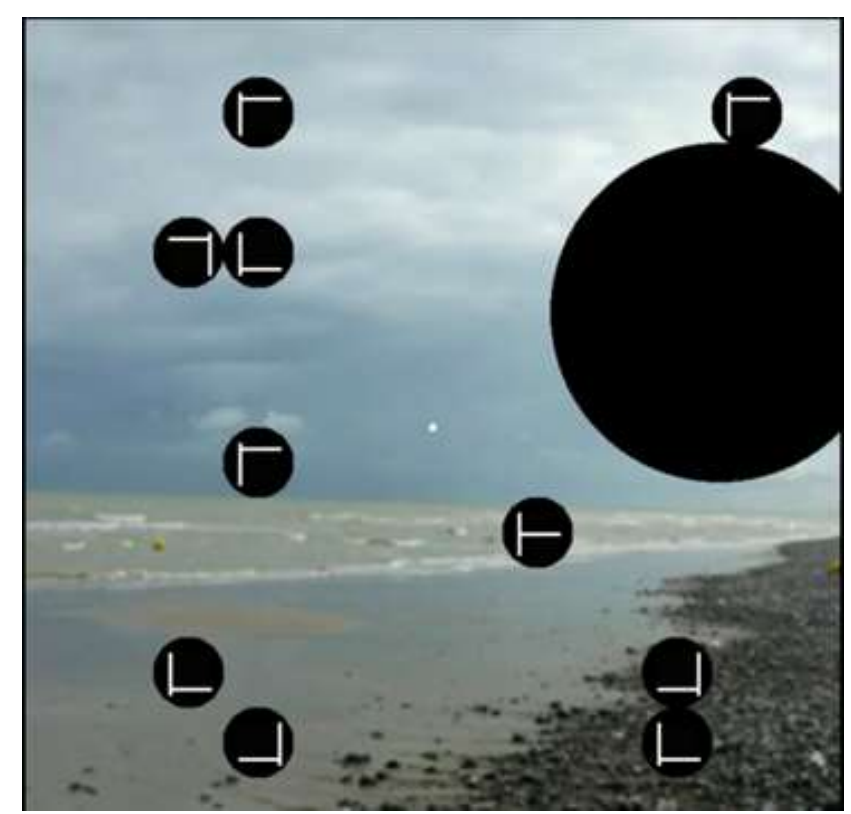

Figure 1. A sample search array from a scotoma search trial. Participants searched for a T presented among L-shaped distractors and reported the target's orientation. The scotoma was a $6.7^{\circ}$ black circle presented at the participants' fixation location as estimated by the eye tracker, which was updated every screen refresh $(100 \mathrm{~Hz})$. Letters were displayed against task-irrelevant natural scenes to increase the realistic nature of the search task. A video depicting several trials is available at https://osf.io/4g7vc/.

Design. After a 36-trial practice block with the scotoma and equal probabilities of targets appearing in all locations, participants completed 16 blocks, each with 36 search trials, for a total of 576 trials.

Training. The first 8 blocks were training blocks, which contained the targets more often in one high-probability "rich" quadrant as opposed to the other three low-probability "sparse" quadrants. Specifically, in each training block, the rich quadrant contained the target on half of trials (18/36 trials), and each of the remaining sparse quadrants contained the target on 1/6 of trials (6/36 trials). The rich quadrant was counterbalanced across participants, who were not informed of the target's location probability.

In Experiment 1, training was conducted without the central scotoma. In Experiment 2, training was conducted with a central scotoma on all trials.

Testing. The last 8 blocks were testing blocks, which alternated between blocks with and without the scotoma. Vision status - no-scotoma or scotoma - of the first testing block was counterbalanced across participants. In the testing phase, the targets were presented equally often in each quadrant ( 9 trials per block contained a target in each quadrant). The same testing conditions were used in Experiments 1 and 2. Figure 2 illustrates the block structure used in the two experiments, for training and testing phases separately. 


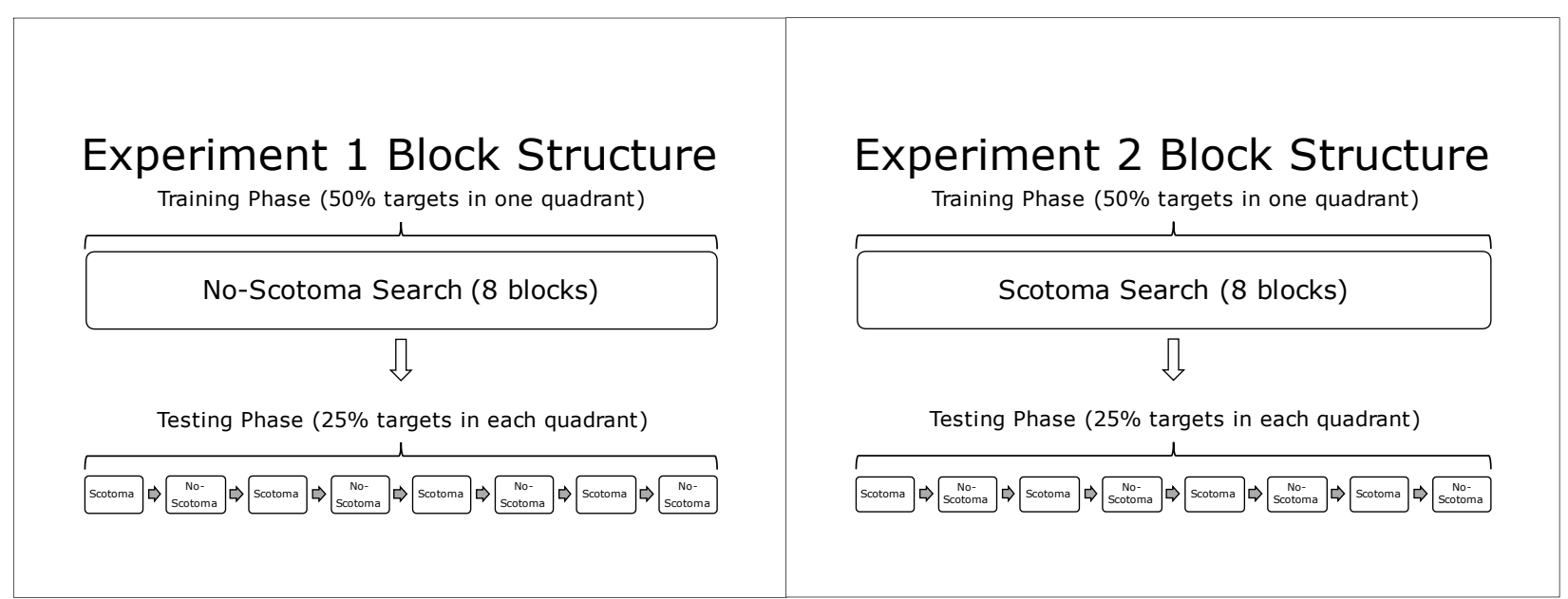

Figure 2. Block structure of Experiment 1 (left) and Experiment 2 (right). The training phase contained 8 blocks of 36 search trials each, either without the central scotoma (Experiment 1) or with the central scotoma (Experiment 2). The target appeared in one, high-probability "rich" quadrant on $50 \%$ of the trials in the training phase. In both experiments, the testing phase contained 4 scotoma blocks and 4 no-scotoma blocks which alternated from block to block, with the order counterbalanced across participants. Testing blocks of both types contained targets equally often in all locations.

Procedure. In both vision status conditions, participants began each trial by pressing the spacebar while looking at a central fixation point; if the eye tracker verified their central fixation, the stimuli (and, if present, the scotoma) then appeared on the screen for 10 seconds or until participants made a response, whichever happened earlier. Participants searched for a letter ' $\mathrm{T}$ ' among 11 'L's and reported the target's orientation $\left(0^{\circ}, 90^{\circ}, 180^{\circ}\right.$, or $\left.270^{\circ}\right)$ using the arrow keys as quickly and accurately as possible. Accuracy feedback - three rising tones for a correct response and a single lower tone for an incorrect response - followed each trial.

Recognition test. Participants answered two questions after the search task to gauge their awareness of the target's location probability. The questions were "Was the T equally likely to appear anywhere on the screen?" and "If you had to choose one quadrant you feel the target T occurred most often, which would you choose?" The first question was a subjective test, and the second question an objective test of awareness. We classified participants as "aware" if they correctly answered the second question, and "unaware" if they responded incorrectly to that question.

Analysis. To incorporate both accuracy and RT in a single measure of performance, we adopted the use of a common measure of adjusted RT known as "inverse efficiency" (Akhtar \& Enns, 1989; Christie \& Klein, 1995; Townsend \& Ashby, 1978, 1983), computed as RT divided by accuracy. Trials with an incorrect response were excluded. The adjusted RT increased RT more for conditions that had lower accuracy. Data reported here are based on the adjusted RT. The pattern of results was qualitatively similar when RT was unadjusted. The Supplementary Materials contain data about mean accuracy as well as mean unadjusted RT. Raw data files and analysis scripts are available at https://osf.io/4g7vc/.

For eye movements, we used the proportion of first fixations landing in the highprobability, "rich" quadrant versus other quadrants as the key index of oculomotor biases. Past research shows that location probability learning increases the proportion of first fixations to the 
rich quadrant - this is not due to reinforcement of first fixations themselves (because participants are not more likely to direct their first fixation to the target than any other location in letter search), but to reinforcement of where targets are ultimately found (Jiang, Won, \& Swallow, 2014). Only fixations occurring more than 100 milliseconds following stimulus onset were considered in these analyses, to ensure that only the first fixation in response to the search array onset was considered. To ensure there was a first fixation on each trial, we also removed trials without any saccades from analyses of adjusted RT and first fixations.

To analyze the time course of learning with adequate statistical power per data point, we grouped every 2 blocks into an epoch: Epochs 1-4 were the no-scotoma training phase; Epochs 5-6 were the testing phase, containing the scotoma and no-scotoma conditions. For convenience, we refer to Epochs 5 and 6 in both scotoma and no-scotoma conditions even though vision status alternated by block with order counterbalanced.

\section{Results}

\section{Acquisition of Probability Learning: Training phase adjusted RT 1.1 Data from all participants}

In the training phase, the target more often appeared in a high-probability "rich" quadrant than in any of the other three "sparse" quadrants. As shown in Figure 3, participants acquired a spatial attentional preference for the high-probability quadrant, both when training was conducted without the central scotoma (Experiment 1) and with it (Experiment 2). In Experiment 1, an ANOVA on target quadrant (rich and sparse) and epoch (1-4) showed significantly lower adjusted RT in the rich than the sparse quadrants, $F(1,23)=36.06, p<.001, \eta_{p}{ }^{2}=.61$. Performance was better in later epochs than earlier ones, $F(3,69)=14.99, p<.001, \eta_{p}{ }^{2}=.40$. The two factors did not interact significantly, $F(3,69)=1.66, p=.18, \eta_{p}{ }^{2}=.07$. The pattern of results was similar in Experiment 2, when training was conducted with the central scotoma. The main effects of target quadrant, $\left[F(1,23)=5.50, p=.028, \eta_{p}{ }^{2}=.19\right]$ and epoch $[F(3,69)=$ $11.15, p<.001, \eta_{p}{ }^{2}=.33$ ] were both significant, without an interaction, $F<1$.

A direct comparison between the two experiments using experiment as a between-subject factor and target quadrant and epoch as within-subject factors showed that participants were significantly slower when trained with the central scotoma than without it, $F(1,46)=54.05, p$ $<.001, \eta_{p}{ }^{2}=.54$. However, the size of location probability learning was comparable between the two experiments, $F<1$ for the interaction between target quadrant and experiment. 


\section{Exp. 1: All participants \\ $(n=24)$}

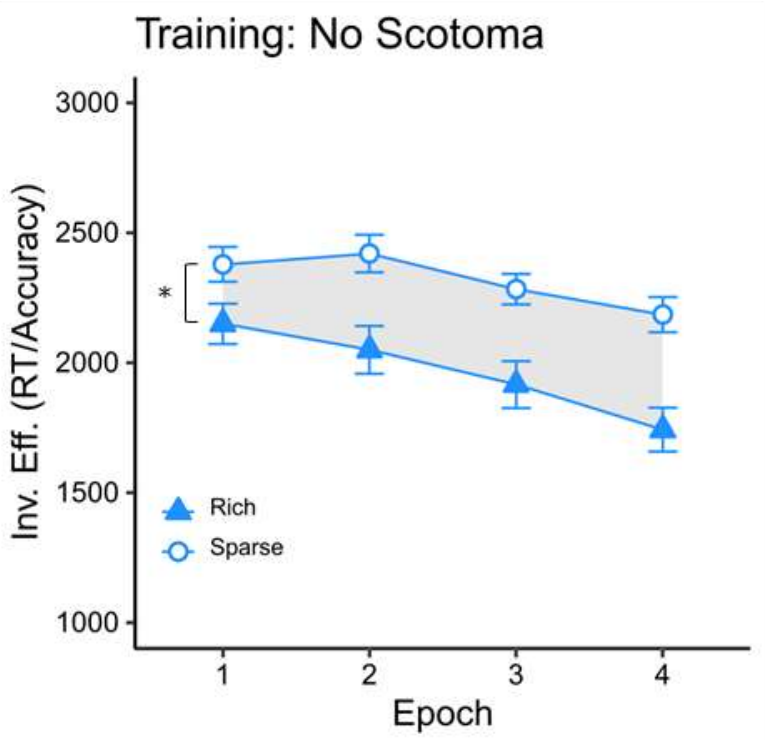

\section{Exp. 2: All participants}

$(\mathbf{n}=24)$

Training: Scotoma

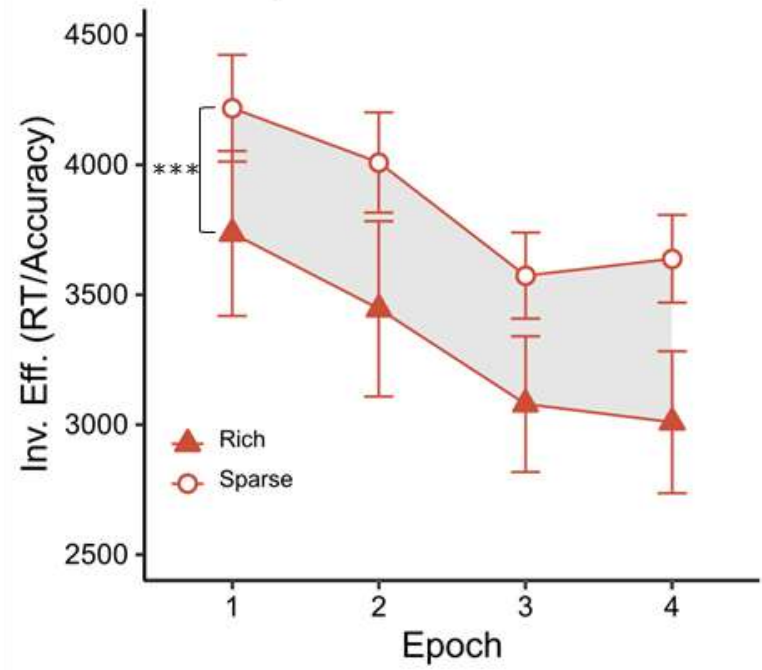

Figure 3. Adjusted RT in the training phase from all participants. Every two blocks (i.e., 72 trials) were grouped into an epoch to reduce variation due to statistical noise. Left: training without the central scotoma (Experiment 1). Right: training with the central scotoma (Experiment 2). Error bars show +/-1 S.E. of the mean. Asterisks denote significance of the main effect of target quadrant. $* p<.05 ;{ }^{* *} p<.01 ; * * * p<.001$; ns: not significant.

\subsection{Effects of explicit awareness}

The analysis reported above showed apparently comparable location probability learning when trained without and with the central scotoma. However, that analysis combined data across all participants, regardless of whether they showed explicit awareness of the target's location probability. That is, were the effects of simulated scotomas equivalent in participants who did and did not report awareness of the target's location probability? To address this question, we separated the training data depending on whether participants correctly recognized the rich quadrant ("aware" participants) or not ("unaware" participants). There were 10 aware and 14 unaware participants in Experiment 1, and 11 aware and 13 unaware participants in Experiment 2. 
Exp. 1

Aware $(n=10)$

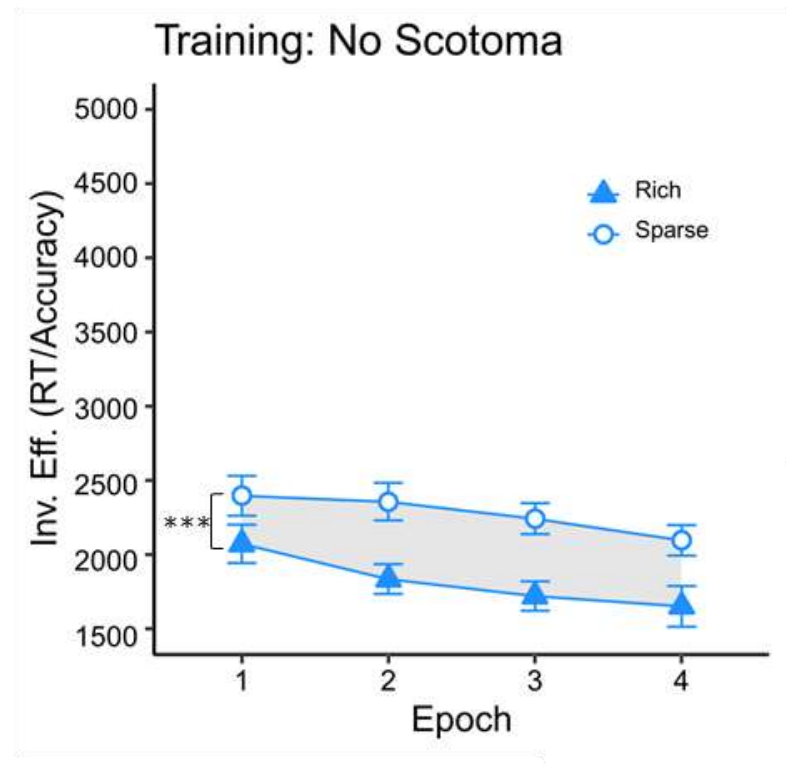

Exp. 1

Unaware $(n=14)$

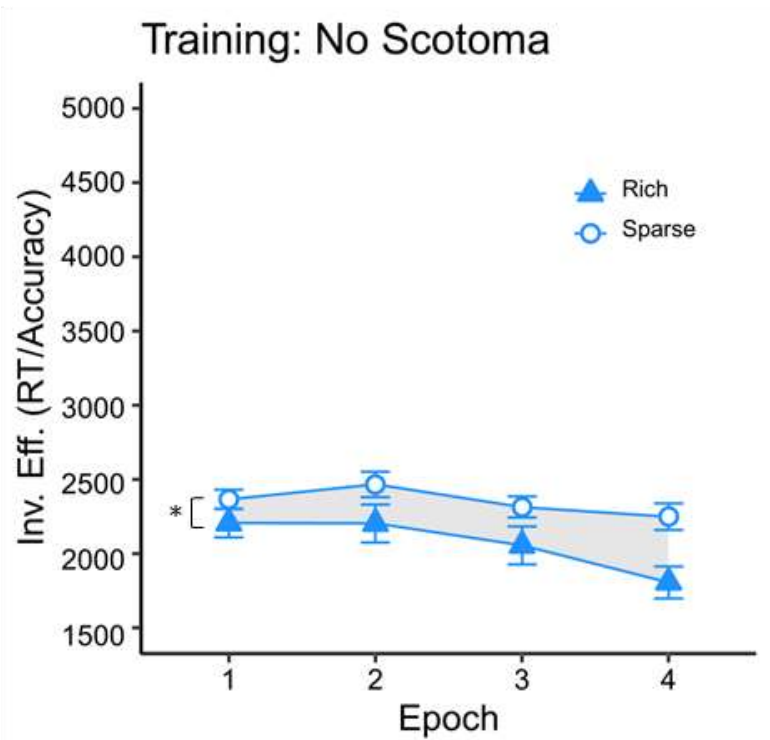

Exp. 2

Aware $(n=11)$

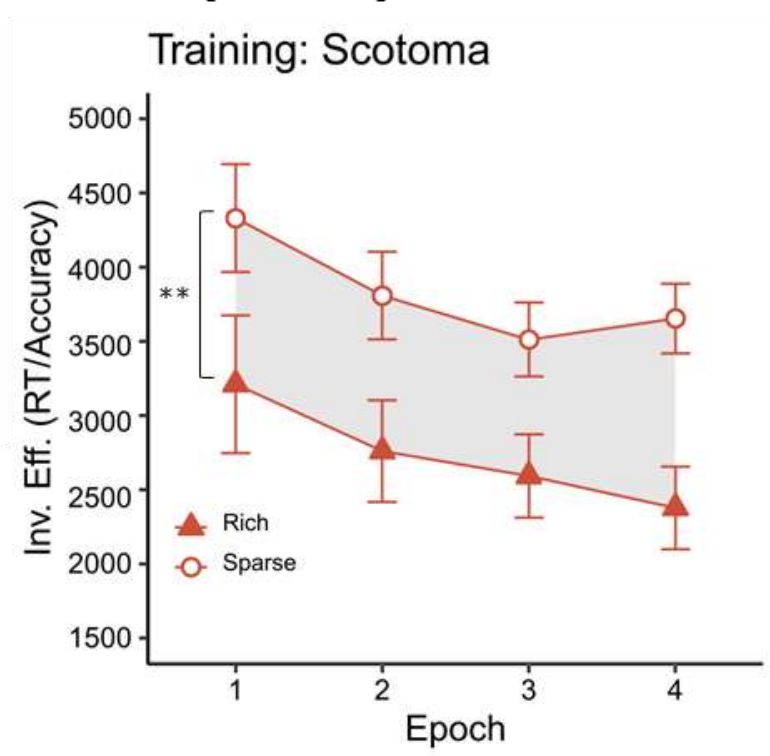

Figure 4. Adjusted RT in the training phase, separately for participants who did or did not have awareness of the target's location probability. Top Left: Training data from 10 aware participants in Experiment 1, who completed training with no central scotoma. Bottom Left: Training data from 14 unaware participants in Experiment 1, who completed training with no central scotoma. Top Right: Training data from 11 aware participants in Experiment 2, who completed training with the central scotoma. Bottom Right: Training data from 13 unaware participants in Experiment 2, who completed search with the central scotoma. Error bars show +/-1 S.E. of the mean. $* p<.05 ; * * p<.01 ; * * * p<.001$; ns: not significant. 
As shown in Figure 4, awareness had different effects on location probability learning depending on whether training was conducted with or without a central scotoma. When trained with no scotoma, both aware and unaware participants acquired location probability learning. In Experiment 1, an ANOVA using awareness as a between-subject factor and target quadrant (rich vs. sparse) and training phase epoch (1-4) as within-subject factors revealed just a significant main effect of target quadrant, $F(1,22)=37.94, p<.001, \eta_{p}{ }^{2}=.63$, an effect that did not interact with awareness, $F(1,22)=2.19, p=.152, \eta_{p}{ }^{2}=.09$. Follow-up tests confirmed that probability learning was significant both for aware participants, $F(1,9)=34.31, p<.001, \eta_{p}{ }^{2}=.47$, and for unaware participants, $F(1,13)=12.05, p=.004, \eta_{p}{ }^{2}=.48$. In contrast, when trained with a central scotoma, probability learning was only observed for aware participants. In Experiment 2, the significant main effect of target quadrant $\left[F(1,22)=6.64, p=.017, \eta_{p}{ }^{2}=.23\right]$ was qualified by a significant interaction between target quadrant and awareness, $F(1,22)=5.76, p=.025, \eta_{p}{ }^{2}$ $=.21$ and no significant main effect of awareness, $F(1,22)=2.39, p=.137$. Whereas aware participants in Experiment 2 showed probability learning, $F(1,10)=14.41, p=.004, \eta_{p}{ }^{2}=.59$, unaware participants did not, $F<1$.

Since there was a numerical advantage for the rich quadrant in Experiment 2's unaware participants, we assessed evidence in favor of the null effect of target quadrant using a Bayesian ANOVA on adjusted RT with epoch and target quadrant as fixed factors and participant ID as a random factor (Morey et al., 2015). The preferred model was one with only epoch, $\mathrm{BF}_{10}=4.15$, indicating a preference of this model to the null model by a factor of 4.15 . All other models were less preferred than the null model, including the effect of target quadrant alone, $\mathrm{BF}_{01}=4.44$, indicating a preference for the null model by a factor of 4.44. The Bayesian analysis provided moderate evidence for the lack of an effect of implicit probability learning in unaware participants completing training with simulated CVL.

Thus, whereas no-scotoma search supported location probability learning even in the absence of explicit awareness about the target's location probability learning, this was not the case with a central scotoma. Implicit location probability learning was insignificant when trained with central vision loss. In this condition, learning was driven almost entirely by explicit learning of where the target was likely to be.

\section{Testing phase adjusted RT}

\subsection{Experiment 1}

Experiment 1 showed that without a central scotoma, participants acquired location probability learning regardless of whether they were aware of the target's location probability. Can they implement the successful learning when tested with a central scotoma? As in the training data, awareness did not alter the pattern of results in Experiment 1 (see Supplementary Materials). Data reported below were consistent for aware and unaware participants, with adjusted RT not showing a significant effect of awareness or its interaction with target location, $F \mathrm{~s}<1$. 


\section{Exp. 1 \\ All participants $(n=24)$}
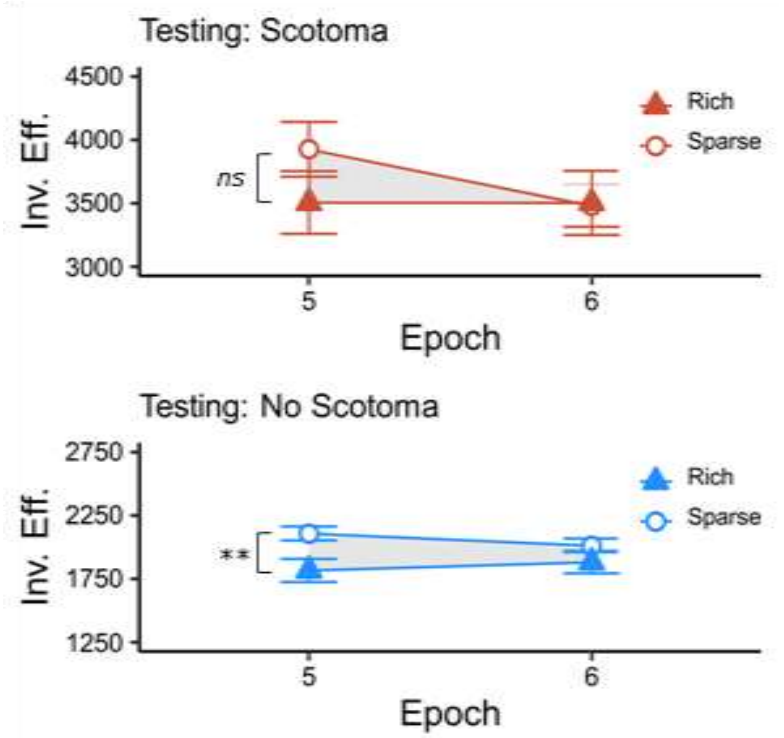

Figure 5. Adjusted RT in the testing phase of Experiment 1. Following training with no central scotoma, participants were tested either with (top) or without a central scotoma (bottom) for two additional epochs. The target's location distribution was even to prevent additional learning. Error bars show +/- 1 S.E. of the mean. ${ }^{*} p<.05$; ** $p<.01$; *** $p<.001$; ns: not significant.

To examine the roles of the central scotoma during testing, we conducted an ANOVA on testing data from Experiment 1 using vision status (no-scotoma and scotoma), testing epoch (5 and 6), and target quadrant (previously rich and sparse) as within-subject factors (Figure 5). Search with a central scotoma slowed down RT substantially, $F(1,23)=104.40, p<.001, \eta_{p}{ }^{2}$ $=.82$, but this did not interact with any other factors, smallest $p=.19$. In this analysis, although the main effect of target quadrant was not significant, $F(1,23)=2.56, p=.12$, this effect interacted with epoch, $F(1,23)=6.81, p=.016, \eta_{p}{ }^{2}=.23$. Follow-up tests showed that in Epoch 5 , participants continued to show location probability learning, $F(1,23)=7.11, p=.014, \eta_{p}{ }^{2}$ $=.24$, an effect that did not interact with vision status, $F<1$. By Epoch 6 , the main effect of target quadrant was no longer significant, $F<1$, an effect that did not interact with vision status, $F<1$. Probability learning appears to have affected search for only the first half of testing in both scotoma and no-scotoma, extinguishing in the second half of testing.

Thus, Experiment 1 showed that following a successful induction of a spatial attentional bias toward a high-probability quadrant, participants continued to prioritize that quadrant for a short time (about 100 trials), but this effect dissipated and became undetectable in the next 100 trials of testing. The pattern of results was statistically indistinguishable between testing blocks with and without the scotoma.

\subsection{Experiment 2}

As shown in the training phase of Experiment 2, training with CVL yielded location probability learning only in participants who were aware of the target's location probability. But was there any evidence of latent learning in unaware participants that might reemerge when tested with no scotoma? In this analysis, we separated aware and unaware participants because 
they demonstrated different patterns of results during Experiment 2's training phase. Figure 6 shows the results.

Exp. 2

Aware $(n=11)$
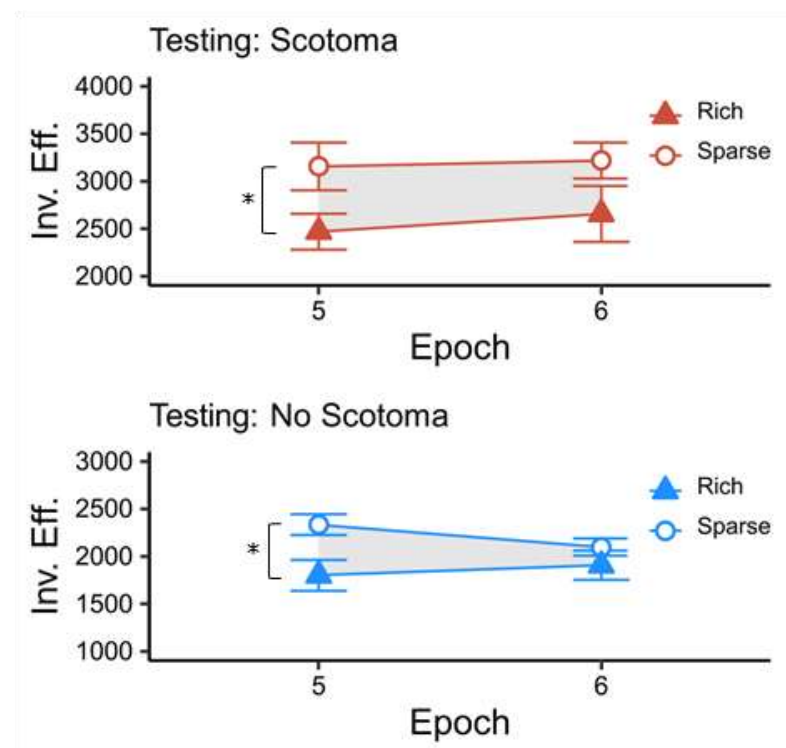

Exp 2. Unaware $(n=13)$
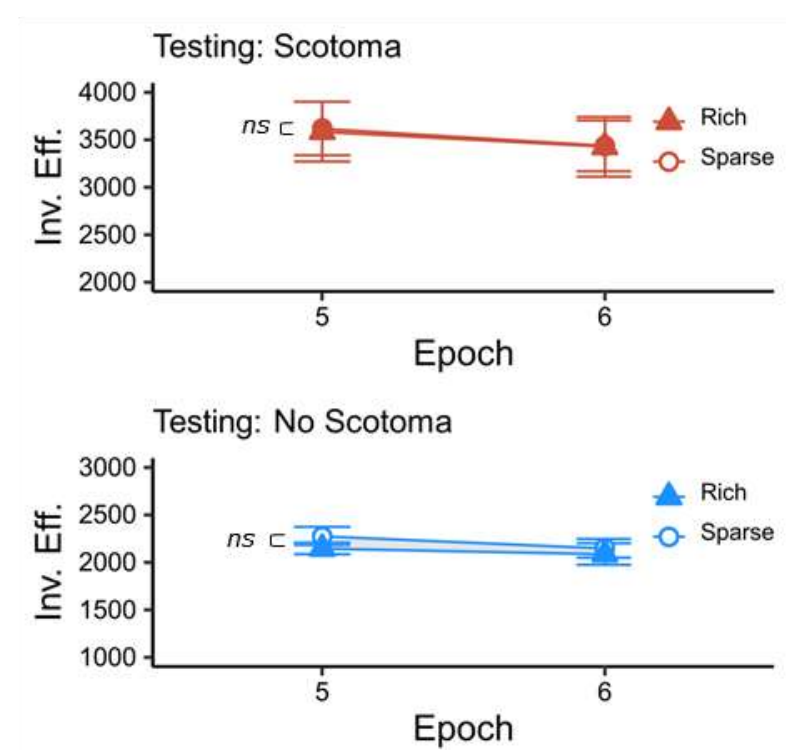

Figure 6. Testing phase adjusted RT in Experiment 2. Top Left: Data from 11 aware participants during testing blocks with the central scotoma. Bottom Left: Data from 11 aware participants during testing blocks with no scotoma. Top Right: Data from 13 unaware participants during testing blocks with the central scotoma. Bottom Right: Data from 13 unaware participants during testing blocks with no scotoma. Error bars show +/- 1 S.E. of the mean. ${ }^{*} p<.05$; $* * p<.01$; $* * * p<.001 ;$ ns: not significant.

(1) Aware participants in Experiment 2

After successfully acquiring probability learning following training with a central scotoma, aware participants continued to demonstrate a spatial preference for the previously high-probability quadrant. This was the case when tested with a central scotoma, $F(1,10)=6.97$, $p=.025, \eta_{p}{ }^{2}=.41$, for the main effect of target quadrant. This effect did not significantly weaken from Epoch 5 to 6 , as there was no interaction between target quadrant and epoch, $F<1$. Learning also persisted without the scotoma, $F(1,10)=7.71, p=.019, \eta_{p}{ }^{2}=.44$, for the main effect of target quadrant. The effect weakened from Epoch 5 to 6, producing an interaction between target quadrant and testing epoch, $F(1,10)=10.31, p<.009, \eta_{p}{ }^{2}=.51$. Overall, aware participants showed a persisting attentional bias toward the previously high-probability quadrant that was present both when tested with and without the central scotoma.

(2) Unaware participants in Experiment 2

In contrast, unaware participants, who evidenced no learning in the training phase of Experiment 2, also did not show any sign of a reemergence of learning in the testing phase. When tested with a central scotoma, they continued to show no adjusted RT benefit in the previously high-probability quadrant, $F<1$. Even with no scotoma, we did not observe any RT benefit, as might have been expected if participants acquired implicit learning but could not express it with a central scotoma. When tested with no scotoma, these participants showed no RT 
difference between the previously rich and sparse quadrants, $F(1,12)=1.64, p=.224$, an effect that did not interact with epoch, $F<1$.

\section{Direction of the first saccadic eye movement}

The first saccadic eye movement in search tasks typically appears within $200 \mathrm{~ms}$ after stimulus onset. It occurs much sooner than the manual response and is a useful index of an early spatial attentional bias (Jiang et al., 2014). Any attentional preference for the high-probability quadrant should result in a tendency to saccade toward that quadrant at levels significantly higher than the $25 \%$ expected by chance. Here we computed the proportion of trials in which the first saccade landed in the high-probability quadrant, averaged within each of 3 conditions: training, no-scotoma testing, and scotoma testing. Because awareness was found to mediate learning as measured by adjusted RT in one of the experiments, this analysis was conducted separately for aware and unaware participants in the two experiments.

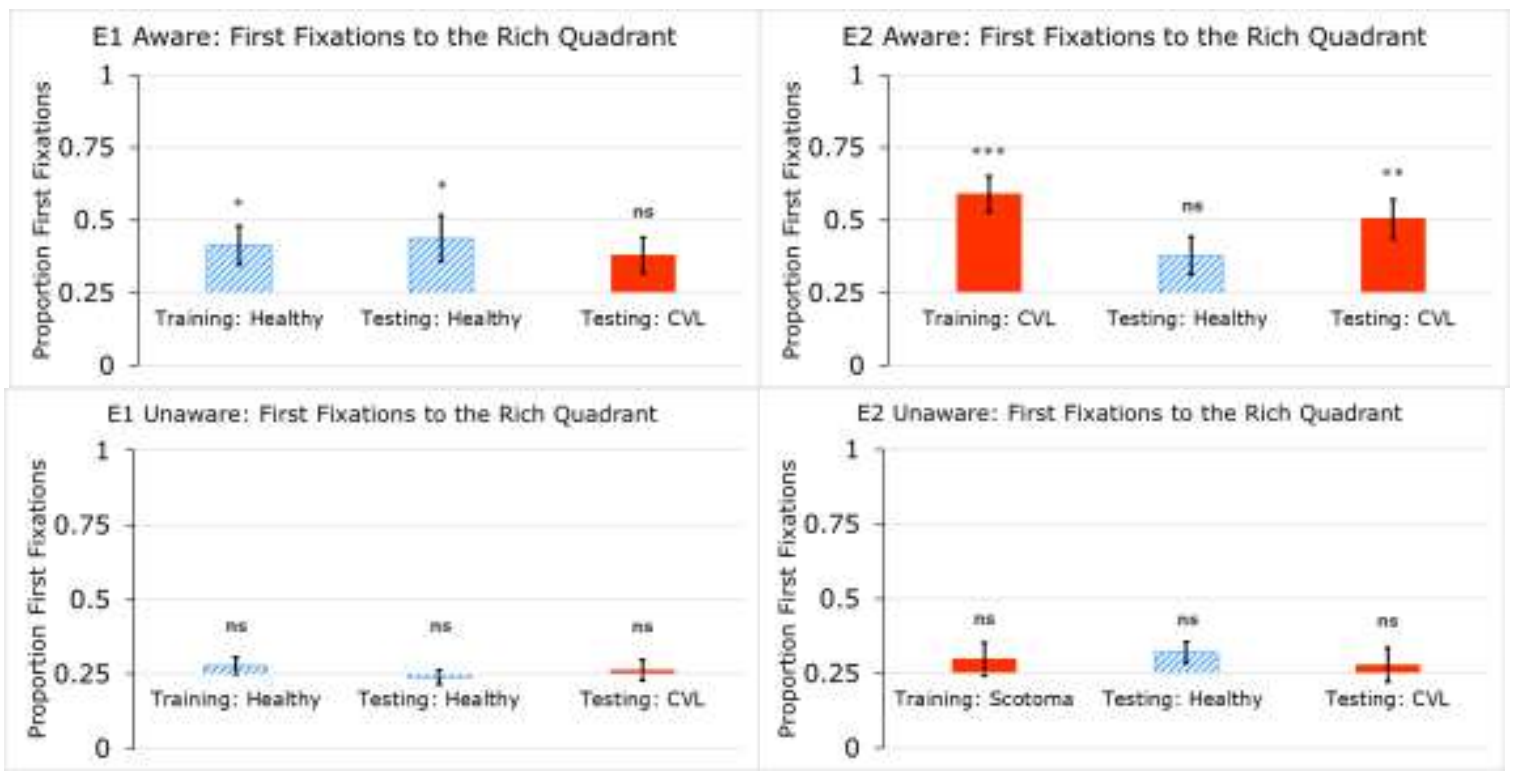

Figure 7. Proportion of first fixations to the rich quadrant in the training phase, healthy testing phase, and CVL testing phase. Left: Experiment 1 (training with healthy vision). Right: Experiment 2 (training with a central scotoma). Chance is $25 \%$ of first fixations in each quadrant. Error bars show +/- 1 S.E. of the mean. * $p<.05$; ** $p<.01$; *** $p<.001$; ns: not significant.

As shown in Figure 7, the first fixation was largely influenced by explicit awareness about the target's location probability. In both experiments, aware participants showed a stronger tendency to saccade toward the rich quadrant, and this was significant in four of the six conditions plotted in Figure 7. The two conditions in which aware participants did not direct first saccades more often to the rich quadrant were when they searched in testing epochs with the untrained vision status, and in both cases there were numerically more first saccades to the rich quadrant. In contrast, unaware participants did not show a clear oculomotor bias toward the rich quadrant in either experiment. Despite not having an initial overt (oculomotor) bias toward the rich quadrant, the unaware participants did acquire implicit learning as measured by adjusted RT when trained with no central scotoma. This is consistent with the possibility that location probability learning reflects changes in visuospatial attention rather than an oculomotor habit. 


\section{Discussion}

In two experiments, we examined the impact of simulated central vision loss on the acquisition and expression of implicit location probability learning. Experiment 1 showed that attentional biases acquired with no scotoma persisted for a short time (about 100 trials) after the target's location probability became unbiased. It then extinguished in the next 100 trials. This pattern of results was independent of participants' awareness of the target's location probability. In addition, it was statistically indistinguishable when tested with or without a central scotoma. These data showed that participants could acquire implicit location probability learning with no central scotoma. In addition, the effect may persist, in a somewhat weakened format, in a testing phase both with and without a central scotoma.

Experiment 2 trained participants with a central scotoma. Results differed from those of Experiment 1. Specifically, Experiment 2 showed a dissociation between aware and unaware participants in the acquisition of location probability learning. Aware participants showed significant learning that persisted during testing regardless of the presence of the scotoma. In contrast, unaware participants did not show evidence of learning in the training phase. Learning also failed to reemerge in the testing phase when the scotoma was removed, suggesting that the implicit spatial learning was never acquired in the first place.

\section{CVL impaired acquisition of implicit location probability learning}

The lack of location probability learning in the unaware participants of Experiment 2 was striking, and contrasts with intact statistical learning in contextual cueing paradigms that simulated CVL (Geringswald et al., 2012; Geringswald, Herbik, et al., 2013). To our knowledge, simulated CVL is one of few conditions impairing probability learning. When searching with no vision loss, location probability learning is an extremely robust effect, with a measured effect size Cohen's $d$ often exceeding 2 (Jiang, Swallow, et al., 2013). The number of unaware participants in Experiment 2 - N of 13 - should have yielded a statistical power greater than $99 \%$ in detecting an effect size of that magnitude at a .05 alpha level. The lack of implicit location probability learning with central scotoma suggests that at the very least, central vision loss weakened implicit location probability learning.

\section{CVL did not eliminate implicitly learned attentional guidance}

Experiment 2 provided clear evidence that central vision loss interfered with the acquisition of implicit location probability learning. Did central vision loss also interfere with the expression of learning, as would be expected if CVL impairs implicit attentional guidance? The answer, from Experiment 1, was a tentative no. If central vision loss interfered with the expression of learning, one might expect location probability learning to disappear in Experiment 1 when participants were tested with a central scotoma. However, the data showed some evidence of a persisting spatial bias, though the effect dissipated over time. The lack of a vision status-by-location interaction in Experiment 1 suggests that the persistence of location probability learning was independent of vision status during test. However, this conclusion was based on a null statistical result. Future studies with a greater sample size are needed to reach definitive conclusions regarding whether the expression of location probability learning is impaired by CVL.

\section{How does awareness affect location probability learning?}

A particularly interesting feature of these results is that, while aware participants showed effects of probability learning regardless of scotoma condition, unaware participants displayed location probability learning only when searching without a scotoma (in Experiment 1). Why did 
the two conditions diverge in their effects of awareness? One possibility is that CVL may interfere more with mechanisms related to conscious goal-driven attention than implicit attentional biases. In this respect, our findings are consistent with work showing that explicit (but not implicit) contextual cueing is intact during search with simulated CVL, albeit smaller than in search without CVL (Pollmann, Geringswald, Wei, \& Porracin, 2020). This possibility is also consistent with recent theories that implicit location probability learning relies on different mechanisms of attentional guidance than goal-driven attention, whether goal-driven attention is induced via task instructions or incidentally through conscious awareness of target probabilities (Jiang, 2018).

\section{First fixations indexed location probability learning only in aware participants}

Eye tracking in these experiments revealed that only explicit location probability learning affected the proportion of first fixations to the target-rich quadrant. In both scotoma and noscotoma search, unaware participants showed no tendency to direct their first saccade towards the rich quadrant. Data from aware participants suggests that CVL, while impairing various elements of oculomotor control as evidenced by other studies (Caldani et al., 2019; Thibaut et al., 2017), did not impair the ability to direct goal-driven attention to the rich quadrant. This is not particularly surprising given the size of a screen quadrant makes it easy to fixate somewhere within the quadrant even if precise direction of saccades is more difficult due to the scotoma.

This is the first report in any population to directly investigate effects of awareness on where participants direct their initial saccades in location probability learning. Previous studies have suggested that implicit location probability learning reflects the acquisition of attentional habits encoded as search vectors relative to fixation rather than enhancing specific locations within priority maps (Jiang \& Sisk, 2019). It has remained unclear whether attentional habits are learned within systems of oculomotor control themselves or earlier stages of attentional control. That unaware participants show no bias in directing first saccades to the rich quadrant suggests that learning does not reflect oculomotor learning. This possibility is further supported by the results from Experiment 1 suggesting that attentional guidance in location probability learning may be intact during simulated scotoma search. Given known impairments to oculomotor control from simulated CVL that may prevent accurate calibration of eye movements in probability learning paradigms (Caldani et al., 2019; Thibaut et al., 2017), it seems likely that, if probability is a form of oculomotor learning, it would be impaired by simulated CVL. This is consistent with the premotor theory of attention (Rizzolatti, Riggio, Dascola, \& Umiltá, 1987). Future research is needed to further clarify the specific mechanisms of learned attentional control.

\section{Relationship to clinical central vision loss}

The use of simulated CVL rather than clinical populations raises important questions about the applicability of the present results to people who have CVL. There is evidence that simulated CVL provides an accurate substitution for at least some aspects of clinical CVL. Like CVL in patients with conditions like macular degeneration (Caldani et al., 2019; Crabb \& Taylor, 2017; Thibaut et al., 2017), simulated CVL increases reaction times and fixation duration during visual search (Bertera, 1988; Cornelissen, Bruin, \& Kooijman, 2005), though this may depend on specific parameters of the task and the simulated scotoma (Geringswald et al., 2012; Shen et al., 2003). Studies also showed impairments in contextual cueing in a clinical population with CVL that were analogous to those found in simulated studies (Geringswald et al., 2013). It is plausible, however, that clinical CVL populations may be more likely to acquire implicit location probability learning than healthy participants in simulated conditions; their extensive experience with central scotomas typically results in their use of preferred retinal loci (PRLs), 
potentially allowing attentional habits to emerge relative to those loci rather than the fovea. In contrast, this process might not occur in naïve participants searching with simulated CVL, for whom development of relatively stable PRLs takes many hours of training (Kwon et al., 2013). Furthermore, despite most cases of CVL arising in older adults, previous research has demonstrated that healthy aging alone does not impair location probability learning (Twedell, Koutstaal, \& Jiang, 2016). As such, we think research on the effects of clinical CVL on implicit location probability learning would be a valuable direction for future research with important implications for our understanding of attentional learning as well as CVL itself.

\section{Conclusion}

The present study investigated the effects of simulated central vision loss on implicit attentional learning in a location probability learning paradigm. Our results provide evidence that simulated central vision loss impairs the acquisition of implicitly learned attentional biases but not necessarily attentional guidance itself. These results suggest that implicit location probability learning involves attentional guidance via search habits rather than via the priority maps involved in goal-driven and stimulus-driven attention. They furthermore suggest that these search habits may not operate directly via oculomotor control but via related, earlier mechanisms of attentional control. They also provide valuable context for future research on these implicit attentional learning mechanisms in clinical populations with central vision loss, guiding the development of learning contexts in which implicit attention is intact and can be used in training attentional learning in real-world situations for people with central vision loss.

\section{Acknowledgements}

This research was supported in part by the University of Minnesota Engdahl Research fund, a University of Minnesota CLA Doctoral Dissertation Fellowship, and the NSF NRT Fellowship DGE-17348915. We thank Julie Jia, Hunter Schouviller, Harlequin Mao, and Carolyn Henkle for assistance with data collection.

Correspondence concerning this article should be addressed to Douglas

A. Addleman, HB 6207, Moore Hall, Department of Psychological and Brain Sciences, Dartmouth College, Hanover, NH 03755. E-mail: daddleman@dartmouth.edu

\section{References}

Akhtar, N., \& Enns, J. T. (1989). Relations between covert orienting and filtering in the development of visual attention. Journal of Experimental Child Psychology, 48(2), 315334. https://doi.org/10.1016/0022-0965(89)90008-8

Awh, E., Belopolsky, A. V., \& Theeuwes, J. (2012). Top-down versus bottom-up attentional control: A failed theoretical dichotomy. Trends in Cognitive Sciences, 16(8), 437-443. https://doi.org/10.1016/j.tics.2012.06.010

Bertera, J. H. (1988). The effect of simulated scotomas on visual search in normal subjects. Investigative Ophthalmology \& Visual Science, 29(3), 470-475.

Caldani, S., Chatard, H., Wiener-Vacher, S., \& Bucci, M. P. (2019). Visual searching capabilities in Age-Related Macular Degeneration (AMD) subjects. Applied Neuropsychology: Adult, 1-8. https://doi.org/10.1080/23279095.2019.1678158

Cheung, S. H., \& Legge, G. E. (2005). Functional and cortical adaptations to central vision loss. Visual Neuroscience, 22(2), 187-201. https://doi.org/10.1017/S0952523805222071 
Christie, J., \& Klein, R. (1995). Familiarity and attention: Does what we know affect what we notice? Memory \& Cognition, 23(5), 547-550.

Chun, M. M., \& Jiang, Y. (1998). Contextual cueing: Implicit learning and memory of visual context guides spatial attention. Cognitive Psychology, 36(1), 28-71. https://doi.org/10.1006/cogp.1998.0681

Colagiuri, B., \& Livesey, E. J. (2016). Contextual cuing as a form of nonconscious learning: Theoretical and empirical analysis in large and very large samples. Psychonomic Bulletin \& Review, 23, 1996-2009. https://doi.org/10.3758/s13423-016-1063-0

Cornelissen, F. W., Bruin, K. J., \& Kooijman, A. C. (2005). The influence of artificial scotomas on eye movements during visual search. Optometry and Vision Science, 82(1), 27-35. https://doi.org/10.1097/01.OPX.0000150250.14720.C5

Crabb, D. P., \& Taylor, D. J. (2017). Searching for unity: Real-world versus item-based visual search in age-related eye disease. Behavioral and Brain Sciences, 40(e135). https://doi.org/10.1017/S0140525X16000054

Druker, M., \& Anderson, B. (2010). Spatial probability aids visual stimulus discrimination. Frontiers in Human Neuroscience, 4, Article 63. https://doi.org/10.3389/fnhum.2010.00063

Geng, J. J., \& Behrmann, M. (2002). Probability Cuing of Target Location Facilitates Visual Search Implicitly in Normal Participants and Patients with Hemispatial Neglect. Psychological Science, 13(6), 520-525. https://doi.org/10.1111/1467-9280.00491

Geringswald, F., Baumgartner, F. J., \& Pollmann, S. (2013). A behavioral task for the validation of a gaze-contingent simulated scotoma. Behavior Research Methods, 45(4), 1313-1321. https://doi.org/10.3758/s13428-013-0321-6

Geringswald, F., Baumgartner, F., \& Pollmann, S. (2012). Simulated loss of foveal vision eliminates visual search advantage in repeated displays. Frontiers in Human Neuroscience, 6(MAY 2012), 1-9. https://doi.org/10.3389/fnhum.2012.00134

Geringswald, F., Herbik, A., Hoffmann, M. B., \& Pollmann, S. (2013). Contextual cueing impairment in patients with age-related macular degeneration. Journal of Vision, 13(3), 2828. https://doi.org/10.1167/13.3.28

Geringswald, F., \& Pollmann, S. (2015). Central and peripheral vision loss differentially affects contextual cueing in visual search. Journal of Experimental Psychology: Learning Memory and Cognition, 41(5), 1485-1496. https://doi.org/10.1037/xlm0000117

Jiang, Y. V. (2018). Habitual versus goal-driven attention. Cortex, 102, 107-120. https://doi.org/10.1016/j.cortex.2017.06.018

Jiang, Y. V., Capistrano, C. G., Esler, A. N., \& Swallow, K. M. (2013). Directing attention based on incidental learning in children with autism spectrum disorder. Neuropsychology, 27(2), 161-169. https://doi.org/10.1037/a0031648

Jiang, Y. V., Swallow, K. M., Rosenbaum, G. M., \& Herzig, C. (2013). Rapid acquisition but slow extinction of an attentional bias in space. Journal of Experimental Psychology: Human Perception and Performance, 39(1), 87-99. https://doi.org/10.1037/a0027611

Jiang, Y. V, Sha, L. Z., \& Sisk, C. A. (2018). Experience-guided attention: Uniform and implicit. Attention, Perception, \& Psychophysics, 80, 1647-1653. https://doi.org/10.3758/s13414018-1585-9

Jiang, Y. V, \& Sisk, C. A. (2019). Habit-like attention. Current Opinion in Psychology, 29, 6570. https://doi.org/10.1016/j.copsyc.2018.11.014

Jiang, Y. V, Sisk, C. A., \& Toh, Y. N. (2019). Implicit guidance of attention in contextual cueing: Neuropsychological and developmental evidence. Neuroscience \& Biobehavioral 
Reviews, 105.

Jiang, Y. V, Won, B., \& Swallow, K. M. (2014). First saccadic eye movement reveals persistent attentional guidance by implicit learning. Journal of Experimental Psychology: Human Perception \& Performance, 40(3), 1161-1173.

Kleiner, M., Brainard, D., \& Pelli, D. (2007). What's new in Psychtoolbox-3?

Kwon, M., Nandy, A. S., \& Tjan, B. S. (2013). Rapid and persistent adaptability of human oculomotor control in response to simulated central vision loss. Current Biology, 23(17), 1663-1669. https://doi.org/10.1016/j.cub.2013.06.056

Miller, J. (1988). Components of the location probability effect in visual search tasks. Journal of Experimental Psychology: Human Perception and Performance, 14(3), 453-471. https://doi.org/10.1037/0096-1523.14.3.453

Morey, R., Rouder, J. N., Jamil, T., Urbanek, S., Forner, K., \& Ly, A. (2015). BayesFactor. Retrieved from https://richarddmorey.github.io/BayesFactor/

Pennington, K. L., \& Deangelis, M. M. (2016). Epidemiology of age-related macular degeneration (AMD): associations with cardiovascular disease phenotypes and lipid factors. Eye and Vision, 1-20. https://doi.org/10.1186/s40662-016-0063-5

Pollmann, S., Geringswald, F., Wei, P., \& Porracin, E. (2020). Intact contextual cueing for search in realistic scenes with simulated central or peripheral vision loss. Translational Vision Science \& Technology, 9(15), 1-11.

Research, S. (n.d.). EyeLink 1000 Plus. Retrieved from https://www.sr-research.com/eyelink1000-plus/

Rizzolatti, G., Riggio, L., Dascola, I., \& Umiltá, C. (1987). Reorienting attention across the horizontal and vertical meridians: Evidence in favor of a premotor theory of attention. Neuropsychologia, 25(1), 31-40. https://doi.org/10.1016/0028-3932(87)90041-8

Shen, J., Reingold, E. M., Pomplun, M., \& Williams, D. E. (2003). Saccadic selectivity during visual search: The influence of central processing difficulty. In J. Hyona, R. Radach, \& H. Deubel (Eds.), The Mind's Eye: Cognitive and Applied Aspects of Eye Movement Research (pp. 65-88).

Sisk, C. A., Twedell, E. L., Koutstaal, W., Cooper, S. E., \& Jiang, Y. V. (2018). Implicitlylearned spatial attention is unimpaired in patients with Parkinson's disease. Neuropsychologia, 119, 34-44. https://doi.org/10.1016/j.neuropsychologia.2018.07.030

Thibaut, M., Tran, T. H. C., Szaffarczyk, S., \& Boucart, M. (2017). Impact of age-related macular degeneration on object searches in realistic panoramic scenes. Clinical and Experimental Optometry, 1-8. https://doi.org/10.1111/cxo.12644

Townsend, J., \& Ashby, F. (1978). Methods of modeling capacity in simple processing systems. In N. J. J. Castellan \& F. Restle (Eds.), Cognitive theory (vol. 3) (pp. 199-239). https://doi.org/10.1163/_q3_SIM_00374

Townsend, J., \& Ashby, F. (1983). Stochastic modeling of elementary psychological processes. The American Journal of Psychology, 98(3), 480. https://doi.org/10.2307/1422636

Twedell, E., Koutstaal, W., \& Jiang, Y. V. (2016). Aging affects the balance between goalguided and habitual spatial attention. Psychnomic Bulletin \& Review, 1-7. https://doi.org/10.3758/s13423-016-1214-3

Twedell, E. L., Koutstaal, W., \& Jiang, Y. V. (2017). Aging affects the balance between goalguided and habitual spatial attention. Psychonomic Bulletin and Review, 24(4), 1135-1141. https://doi.org/10.3758/s13423-016-1214-3

Won, B., \& Jiang, Y. V. (2015). Spatial working memory interferes with explicit, but not 
probabilistic cuing of spatial attention. Journal of Experimental Psychology: Learning, Memory and Cognition, 41(3), 1-30. https://doi.org/10.1037/xlm0000040

Xiao, J., Hays, J., Ehinger, K. A., Oliva, A., \& Torralba, A. (2010). SUN database: Large-scale scene recognition from abbey to zoo. 2010 IEEE Computer Society Conference on Computer Vision and Pattern Recognition, 3485-3492. https://doi.org/10.1109/CVPR.2010.5539970 